\title{
Density of states approach to the hexagonal Hubbard model at finite density
}

\author{
Michael Körner, ${ }^{1}$ Kurt Langfeld $\odot,{ }^{2}$ Dominik Smith $\odot,{ }^{1}$ and Lorenz von Smekal ${ }^{1}$ \\ ${ }^{1}$ Institut für Theoretische Physik, Justus-Liebig-Universität, \\ Heinrich-Buff-Ring 16, 35392 Giessen, Germany \\ ${ }^{2}$ School of Mathematics, University of Leeds, Leeds LS2 9JT, United Kingdom
}

(Received 15 June 2020; accepted 14 August 2020; published 8 September 2020)

\begin{abstract}
We apply the linear logarithmic relaxation (LLR) method, which generalizes the Wang-Landau algorithm to quantum systems with continuous degrees of freedom, to the fermionic Hubbard model with repulsive interactions on the honeycomb lattice. We compute the generalized density of states of the average Hubbard field and devise two reconstruction schemes to extract physical observables from this result. By computing the particle density as a function of chemical potential we assess the utility of LLR in dealing with the sign problem of this model, which arises away from half filling. We show that the relative advantage over brute-force reweighting grows as the interaction strength is increased and discuss possible future improvements.
\end{abstract}

DOI: $10.1103 /$ PhysRevD.102.054502

\section{INTRODUCTION}

Monte Carlo simulations based on path-integral quantization are a powerful and widely used tool for the study of strongly coupled quantum systems. They are applied in many different areas of physics, ranging from high-energy physics, where they are employed e.g., to study the phase diagram and particle spectrum of quantum chromodynamics (QCD), to condensed matter physics, where they are used to study strongly correlated electron systems. Quite often, they are the only way to obtain reliable information from first principles. Unfortunately, their applicability is restricted to a very special class of systems, namely those where the path integral exhibits a positive-definite measure which can be interpreted as a probability density. This excludes most fermionic systems away from half filling (unless the complex parts of the measure cancel exactly due to an antiunitary symmetry) as well as quantum systems which evolve in real (as opposed to Euclidean) time. This restriction is known as the sign problem and is a longstanding problem of numerical physics.

In principle, brute-force reweighting techniques can be used to extract information about systems with charge imbalance from simulations of a corresponding system at charge neutrality. These are plagued by a severe signal-tonoise ratio problem however, originating from a loss of overlap between the ensembles at zero and nonzero charge

Published by the American Physical Society under the terms of the Creative Commons Attribution 4.0 International license. Further distribution of this work must maintain attribution to the author(s) and the published article's title, journal citation, and DOI. Funded by SCOAP ${ }^{3}$. density when the thermodynamic limit is approached, and typically fail well below $\mu / T \approx 1$ except on very small systems. While a theorem by Troyer and Wiese states that the sign problem is a nondeterministic polynomial hard problem in a generic spin-glass system [1] (making the discovery of a complete universal solution to all sign problems unlikely), many attempts have been made to construct specialized solutions for specific systems (typically by introducing a set of dual variables), or improved general approaches which outperform reweighting, with some success. Most notably, in QCD, Taylor expansions of the partition function with respect to $\mu / T$ have now been pushed to $\mu / T \approx 2$ or 3 [2].

A promising, quite different, idea to deal both with ergodicity problems in Monte Carlo simulations of systems close to a first-order phase transition, and overlap problems resulting from a nonpositive probability measure, is to use non-Markovian random walk simulations, which do not rely on importance sampling with respect to a positive Gibbs factor. A particularly interesting class of algorithms use the inverse density of states as a measure for updating configurations. This measure is positive (semi) definite by definition, and produces a random walk which efficiently samples configuration space even in "deprived" regions with very low probabilistic measure. Prominent examples are the multicanonical algorithm by Berg and Neuhaus [3] and the Wang-Landau approach [4], which both were designed for theories with discrete degrees of freedom.

The linear logarithmic relaxation ("LLR") method, first described in Ref. [5], generalizes this idea to quantum systems with continuous degrees of freedom. The goal of LLR is to estimate the slope $a(X)=\frac{d}{d X} \ln (\rho(X))$, where $X$ 
is some observable and $\rho(X)$ is the "generalized density of states" (GDOS). Once $a(X)$ is obtained, $\rho(X)$ can be reconstructed up to a multiplicative factor by numerical integration and can be used to compute thermodynamic observables. A crucial property of LLR is that it features exponential error suppression: The estimate for $a(X)$, and by extension of $\ln (\rho(X))$, can be obtained with roughly the same statistical error, independent of the exact value of $X$, even if $X$ is from a region of overall low weight.

In recent years, LLR was successfully applied to obtain $\rho(E)$ in $\mathrm{SU}(2)$ and $\mathrm{U}(1)$ [5] as well as $\mathrm{SU}(3)$ [6] gauge theories and was shown to be effective at dealing with ergodicity issues arising at first-order phase transitions in $\mathrm{U}$ (1) gauge theory [7] and the $q=20$ state Potts model [8]. In Ref. [9] LLR was applied to obtain the Polyakov loop distribution for two-color QCD (which has no sign problem) with heavy quarks at finite densities. To deal with the sign problem, one needs to compute the GDOS for the imaginary part of the Euclidean action $\rho\left(S_{I}\right)$, or some related observable. This was achieved using LLR for a $Z_{3}$ spin model at finite charge density [10] and for QCD in the heavy-dense limit [11]. To date, LLR has never been applied to a sign problem of a system with fully dynamical fermions however.

In this work, we apply LLR to the fermionic Hubbard model on the honeycomb lattice away from half filling within a Hybrid Monte Carlo framework. Despite its simplicity, the Hubbard model, which describes fermionic quasiparticles with contact interactions, continues to be of profound interest, as it remains the quintessential example of an interacting fermion system, and can qualitatively describe many nonperturbative phenomena such as dynamical mass-gap generation or superconductivity. On the honeycomb lattice, this model exhibits a second-order phase transition in the universality class of the chiral GrossNeveu model in $2+1$ dimensions [12-15]. With its relativistic dispersion relation for the low-energy excitations in the Dirac-cone region it therefore also provides a convenient lattice regularization, with minimal doubling, of relativistic theories for chiral fermions with local fourfermion interactions such as the Gross-Neveu or NambuJona-Lasinio models which are of continued interest in searches for inhomogeneous phases [16] as predicted also for the QCD phase diagram, mainly from mean-field studies of the Nambu-Jona-Lasinio model [17]. Extended versions of the hexagonal Hubbard model, which include long-range interactions, are also used to realistically describe the physics of both mono- and bilayer graphene $[18,19]$.

Using LLR, here we compute the GDOS for the average of a real-valued auxiliary field, which is introduced in Hybrid Monte Carlo simulations to transmit interelectron interactions. We demonstrate that this result can be used to reconstruct the fermion density as a function of chemical potential. We show that, in its present form, LLR can probe much further into the finite density regime than standard reweighting, that the relative advantage of LLR grows as the interaction strength is increased, and argue that future improvements might put the van Hove singularity in the single-particle bands within reach.

This paper is structured as follows: We start in Sec. II by introducing the basic lattice setup and illustrating the sign problem away from half filling. Subsequently, we introduce the generalized density of states of the average Hubbard field $\rho(s)$ in Sec. III. In Sec. IV, we discuss the reconstruction of the particle density $n$ from $\rho(s)$. We describe two different reconstruction schemes, whereby $n(\mu)$ is obtained from both the canonical and grandcanonical partition functions. As a benchmark, we apply both schemes to test data obtained for the noninteracting tight-binding theory. Full LLR calculations of the interacting theory, including additional numerical details, are then presented in Sec. V. We summarize and conclude in Sec. VI.

\section{LATTICE SETUP AND THE SIGN PROBLEM}

We consider the repulsive Hubbard model on the hexagonal (honeycomb) lattice with fermionic creation operators $\hat{c}_{x}^{\dagger} \equiv\left(\hat{c}_{x, \uparrow}^{\dagger}, \hat{c}_{x, \downarrow}^{\dagger}\right)$ for two spin components at site $x$, which is defined by the effective Hamiltonian for the grand-canonical ensemble:

$$
\begin{aligned}
\hat{\mathcal{H}}= & -\kappa \sum_{\langle x, y\rangle}\left(\hat{c}_{x}^{\dagger} \hat{c}_{y}+\text { H.c. }\right) \\
& +\sum_{x}\left(m_{s} \hat{c}_{x}^{\dagger} \sigma_{3} \hat{c}_{x}+\frac{U}{2} \hat{\rho}_{x}^{2}-\mu \hat{\rho}_{x}\right) .
\end{aligned}
$$

Here $\kappa$ is the hopping parameter, which quantifies the energy cost of fermionic quasiparticles propagating between nearest-neighbor sites. Its phenomenological value in the tight-binding description of the electronic properties of graphene on a substrate is $\kappa \approx 2.7 \mathrm{eV}$. In general, it is typically used to set the energy scale in the Hubbard model. We work in a natural system of units and therefore express all dimensionful quantities in terms of $\kappa$, which effectively corresponds to setting $\kappa \equiv 1$. The sum over $\langle x, y\rangle$ sums all independent pairs of nearest neighbors, $m_{s}$ is the sublattice-staggered mass term (with alternating sign on the two triangular sublattices) for explicit sublattice-symmetry breaking with spin-density-wave order. The chemical potential $\mu$ couples to the charge operator $\hat{\rho}_{x}=\hat{c}_{x}^{\dagger} \hat{c}_{x}-1$ and controls the charge-carrier density. Experimentally this is achieved through chemical doping [20] or electrolytic gate voltages [21], for example. The constant $U$ controls the interaction strength, which is positive in the repulsive Hubbard model. The creation and annihilation operators satisfy the fermionic anticommutation relations $\left\{\hat{c}_{x}, \hat{c}_{y}^{\dagger}\right\}=\delta_{x, y} \mathbb{1}$. Lattice simulations of (1) 
using Hybrid Monte Carlo by now have a long history [22-37]; we thus summarize only the essential steps here. ${ }^{1}$

To derive the functional integral representation of the partition function at inverse temperature $\beta=1 / T$, we first write the exponential in terms of $N_{t}$ identical factors and split the Hamiltonian into the free tight-binding part plus interactions, $\hat{\mathcal{H}}=\hat{\mathcal{H}}_{0}+\hat{\mathcal{H}}_{\text {int }}$. A symmetric Suzuki-Trotter decomposition of each of the factors then yields

$$
\begin{aligned}
\mathcal{Z} & =\operatorname{Tr}\left(e^{-\beta \hat{\mathcal{H}}}\right) \\
& =\operatorname{Tr}\left(e^{-\delta \hat{\mathcal{H}}_{0}} e^{-\delta \hat{\mathcal{H}}_{\text {int }}} e^{-\delta \hat{\mathcal{H}}_{0}} \ldots\right)+O\left(\delta^{2}\right) .
\end{aligned}
$$

This introduces a finite step size $\delta=\beta / N_{t}$ in Euclidean time and a discretization error of $O\left(\delta^{2}\right)$.

As we will see shortly, it is convenient to include the chemical-potential term in the definition of $\hat{\mathcal{H}}_{\text {int }}$ here, i.e., defining

$$
\hat{\mathcal{H}}_{\text {int }} \equiv \sum_{x}\left(\frac{U}{2} \hat{\rho}_{x}^{2}-\mu \hat{\rho}_{x}\right)
$$

The four-fermion interaction in $\hat{\mathcal{H}}_{\text {int }}$ is then converted to bilinears by Hubbard-Stratonovich transformation,

$$
e^{-\delta \hat{\mathcal{H}}_{\mathrm{int}}} \cong \int \mathcal{D} \phi e^{-\frac{\delta}{2 U} \sum_{x} \phi_{x}^{2}} e^{-i \delta \sum_{x}\left(\phi_{x}+i \mu\right) \hat{\rho}_{x}}
$$

whereby the auxiliary ("Hubbard-Coulomb") field $\phi_{x, t}$ is introduced. Finally, the trace over the fermionic operators is performed by integrating the fermionic coherent states [33], which yields

$$
\mathcal{Z}=\int \mathcal{D} \phi \operatorname{det}\left[M(\phi, \mu) M^{\dagger}(\phi,-\mu)\right] \exp \left\{-\frac{\delta}{2 U} \sum_{x, t} \phi_{x, t}^{2}\right\} .
$$

Different versions of the fermion matrix $M(\phi)$ have been used in the past which are either equivalent or at least yield the same continuum limit. In this work we use

$$
\begin{aligned}
M(\phi, \mu)_{(x, t),\left(x^{\prime}, t^{\prime}\right)}= & \delta_{x x^{\prime}} \exp \left\{i \delta\left(\phi_{x, t}+i \mu\right)\right\} \delta_{t t^{\prime}} \\
& -\left(\delta_{x x^{\prime}}-\delta h_{x x^{\prime}}\right) \delta_{t+1, t^{\prime}}, \\
h_{x x^{\prime}}= & \delta_{x x^{\prime}} m_{s}-\kappa \sum_{\vec{n}} \delta_{x^{\prime}, x+\vec{n}},
\end{aligned}
$$

in which the free tight-binding hopping contributions of the form $e^{-\delta h}$ are linearized, in order to be able to work with

\footnotetext{
${ }^{1}$ In particular we omit the discussion of fermionic coherent states and the partial particle-hole transformation that is applied. These and other details can be found e.g., in Refs. [25,33,37].
}

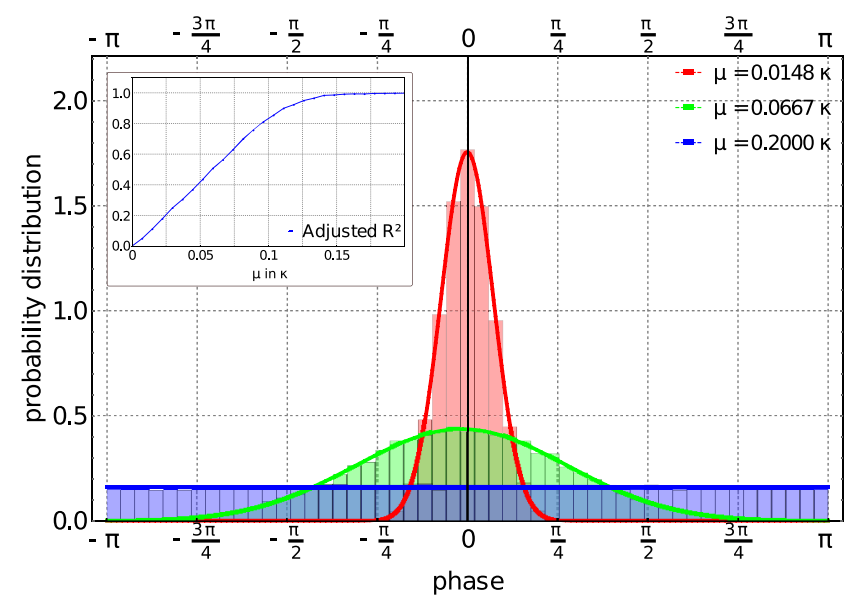

FIG. 1. Histograms of the phase of det $M(\phi, \mu) / \operatorname{det} M(\phi,-\mu)$ for $N_{s}=N_{t}=6, \beta=2.7 \kappa^{-1}, U=\kappa / 2$ at different $\mu$. The results are modeled with Gaussian (for $\mu=0.0148 \kappa$ and $0.0667 \kappa$ ) and uniform (for $\mu=0.2 \kappa$ ) distributions respectively. The inlay shows the adjusted $\mathrm{R}^{2}$ for a constant fit to data at different $\mu$. For $\mu \gtrsim 0.15 \kappa$ the numerical data are well described by a uniform distribution, indicating a hard sign problem. An analogous figure as indication of a sign problem is obtained for graphene with long-range interactions [31].

sparse matrices, but the diagonal couplings to the Hubbard field and chemical potential are not.

It is clear that Eq. (5) is sign problem free at half filling, i.e., for $\mu=0$, as $\operatorname{det}\left(M M^{\dagger}\right)=|\operatorname{det} M|^{2}$. This is no longer true for $\mu \neq 0$. By writing

$$
\begin{aligned}
\mathcal{Z}= & \int \mathcal{D} \phi|\operatorname{det} M(\phi, \mu)|^{2} \frac{\operatorname{det} M(\phi, \mu)}{\operatorname{det} M(\phi,-\mu)} \\
& \times \exp \left\{-\frac{\delta}{2 U} \sum_{t=0}^{N_{t}-1} \sum_{x, y} \phi_{x, t}^{2}\right\},
\end{aligned}
$$

we can consider the complex ratio of determinants with unlike-sign chemical potentials as an observable in the "phase-quenched" theory (defined by the modulus of the fermion determinant) with partition function $\mathcal{Z}_{\mathrm{pq}}$ and obtain

$$
\frac{\mathcal{Z}(\mu)}{\mathcal{Z}_{\mathrm{pq}}(\mu)}=\left\langle\frac{\operatorname{det} M(\phi, \mu)}{\operatorname{det} M(\phi,-\mu)}\right\rangle_{\mathrm{pq}}
$$

This ratio is unity for $\mu \rightarrow 0$ and is a measure of the severity of the sign problem, as it quantifies the effectiveness of brute-force reweighting.

Figure 1 shows histograms of the phase ${ }^{2}$ of the ratio $\operatorname{det} M(\phi, \mu) / \operatorname{det} M(\phi,-\mu)$ for different nonzero values of $\mu$ obtained on a lattice of $N_{s} \times N_{s}$ unit cells, with two sites

\footnotetext{
${ }^{2}$ The modulus also deviates from unity at $\mu \neq 0$ but need not be considered here.
} 
per unit cell, and $N_{s}=N_{t}=6$, at $\beta=2.7 \kappa^{-1}$ and $U=\kappa / 2$, together with fit-model curves. The adjusted $R^{2}$ of a constant fit (corresponding to a uniform distribution) shows a rather rapid crossover and approaches values close to 1 at $\mu \approx 0.15 \kappa$. This indicates that the signal is lost in the noise rather quickly already on small lattices (signaling a hard sign problem), and for rather high temperatures and weak interaction strengths. This effect is enhanced with larger lattice sizes, lower temperatures and larger couplings. To present a quantitative comparison of brute-force reweighting and the LLR method for different system sizes and interaction strengths is one of the main objectives of this work.

\section{GENERALIZED DENSITY OF THE HUBBARD FIELD}

Assume we have a quantum system with action $\beta S(\phi)$. Defining the density of states $\rho(E)$ as

$$
\rho(E)=\int \mathcal{D} \phi \delta(S(\phi)-E)
$$

we can then express the partition function as

$$
\mathcal{Z}=\int d E \rho(E) e^{\beta E}
$$

and the vacuum expectation value of an observable $O(E)$ becomes

$$
\langle O\rangle=\frac{1}{\mathcal{Z}} \int d E O(E) \rho(E) e^{\beta E}
$$

If $\rho(E)$ is known, then $\langle O\rangle$ can be obtained by (numerically or analytically) integrating Eq. (11). Here we have assumed that we know how to express $O$ in terms of $E$, which is in general not the case however. Moreover, Eq. (9) is illdefined if $S(\phi)$ is not strictly real. To compute different observables in a generic setting, the concept of the density of states can thus be generalized to quantities other than the action.

The basic idea of LLR is to obtain $\rho(X)$ (where $X$ is some observable) by carrying out a sequence of "microcanonical" Monte Carlo simulations, in which $X$ is forced to assume a set of different (sufficiently dense) values $X_{i}$. Obtaining the partition function or thermodynamic expectation values then essentially amounts to computing a Fourier or Laplace transform of $\rho(X)$. To alleviate the sign problem, $\rho(X)$ must reflect the phase fluctuations of the path-integral measure. To this end, we consider $\rho(s=\Phi)$ in this work, where $\Phi$ is the spacetime-volume average of the auxiliary field; see below. First, we apply the transformation

$$
\phi_{x, t} \rightarrow \phi_{x, t}-i \mu
$$

to Eq. (5), which then leads to

$$
\begin{aligned}
\mathcal{Z}(\mu) & =\int \mathcal{D} \phi \operatorname{det}\left[M(\phi, \mu) M^{\dagger}(\phi,-\mu)\right] \exp \left\{-\frac{\delta}{2 U} \sum_{x, t} \phi_{x, t}^{2}\right\} \\
& =\int \mathcal{D} \phi|\operatorname{det} M(\phi, 0)|^{2} \exp \left\{-\frac{\delta}{2 U} \sum_{x, t}\left(\phi_{x, t}-i \mu\right)^{2}\right\} .
\end{aligned}
$$

In this formulation, the complex part of the action has been shifted completely to the bosonic sector. Equation (13) is now rewritten as

$$
\begin{aligned}
\mathcal{Z}(\mu)= & \int \mathcal{D} \phi|\operatorname{det} M(\phi, 0)|^{2} \\
& \times \exp \left\{-\frac{\delta}{2 U} \sum_{x, t}\left(\phi_{x, t}-\Phi\right)^{2}-\frac{\delta V}{2 U}(\Phi-i \mu)^{2}\right\},
\end{aligned}
$$

where we have introduced the average Hubbard field

$$
\Phi=\frac{1}{V} \sum_{x, t} \phi_{x, t}, \quad V=2 N_{c} N_{t},
$$

where $N_{c}$ denotes the number of unit cells with two sites each. Finally, we introduce

$$
\begin{aligned}
\rho(s)= & \int \mathcal{D} \phi|\operatorname{det} M(\phi, 0)|^{2} \delta(\Phi-s) \\
& \times \exp \left\{-\frac{\delta}{2 U} \sum_{x, t}\left(\phi_{x, t}-s\right)^{2}\right\},
\end{aligned}
$$

and rewrite the partition function as

$$
\mathcal{Z}(\mu)=\int d s \rho(s) \exp \left\{-\frac{N_{c}}{U T}(s-i \mu)^{2}\right\}
$$

where $\rho(s)$ is the generalized density of states of the average Hubbard field $\Phi$ and will be the target of our LLR calculation.

\section{RECONSTRUCTING THE PARTICLE DENSITY}

Assume we have obtained $\rho(s)$ using some method. Due to the oscillating contribution of the exponential, it is clear that Eq. (17) will be hard, if not impossible, to evaluate numerically. This is exacerbated by the fact that LLR obtains $\rho(s)$ only for a discrete and finite set of points and with finite numerical precision. Our ultimate goal is to obtain the particle density $n(\mu)$. We present two reconstruction schemes which achieve this in the following, which both operate in the frequency domain and avoid the instabilities of Eq. (17). 
We note that $\rho(s)$ has a periodicity of $2 \pi / \beta=2 \pi T$ and can thus be expanded in Fourier series. For later convenience we will first introduce a dimensionless variable and density via

$$
x=\frac{s}{T}, \quad \text { and } \quad \bar{\rho}(x)=T \rho(x T) .
$$

If we furthermore introduce an imaginary chemical potential via

$$
\mu=i \theta T, \quad \text { and } \quad \mathcal{Z}^{I}(\theta) \equiv \mathcal{Z}(i \theta T),
$$

we observe that up to a Gaussian smearing with variance $U / 2 N_{c} T$ the generalized density of states is in fact essentially the same as the partition function at imaginary chemical potential,

$$
\mathcal{Z}^{I}(\theta)=\int d x \bar{\rho}(x) \exp \left\{-\frac{N_{c} T}{U}(x-\theta)^{2}\right\} .
$$

We will obtain $\bar{\rho}(x)$ only at a discrete set of points $x_{n}=2 \pi n / K$, where $n=\{0, \ldots, K-1\}$ and $\bar{\rho}_{n} \equiv \bar{\rho}\left(x_{n}\right)$. We must hence truncate the Fourier series, naturally leading to a discrete Fourier transform which can be used for interpolation via

$$
\tilde{\rho}_{k}=\frac{1}{K} \sum_{n=0}^{K-1} \bar{\rho}_{n} e^{2 \pi i n k / K}, \quad \bar{\rho}(x) \approx \sum_{k=0}^{K-1} \tilde{\rho}_{k} e^{-i k x} .
$$

On the other hand, inserting (21) into (17), we obtain

$$
\begin{aligned}
\mathcal{Z}(\mu) & \approx \int d x\left(\sum_{k=0}^{K-1} \tilde{\rho}_{k} e^{-i k x}\right) \exp \left\{-\frac{N_{c} T}{U}\left(x-i \frac{\mu}{T}\right)^{2}\right\} \\
& =\sqrt{\frac{\pi U}{N_{c} T}} \sum_{k=0}^{K-1} \tilde{\rho}_{k} \exp \left\{-\frac{U}{4 N_{c} T} k^{2}-\frac{\mu}{T} k\right\}
\end{aligned}
$$

and the exact result is recovered for $K \rightarrow \infty$. In fact, in this limit, Eq. (22) becomes the fugacity expansion and we can identify for $k=N$,

$$
Z_{c}(T, N)=\sqrt{\frac{\pi U}{N_{c} T}} \tilde{\rho}_{N} \exp \left\{-\frac{U}{4 N_{c} T} N^{2}\right\}
$$

as the corresponding canonical partition function with particle number $N$. In the infinite-volume limit $N_{c} \rightarrow \infty$ for fixed $N$, or equally so for $T \gg U$, we may therefore neglect the exponential factor and essentially identify the Fourier series coefficients $\tilde{\rho}_{k}$ of our generalized DOS with the canonical partition functions at $N=k$. At the same time, it is also evident from Eq. (22) that the generalized DOS itself then becomes equal, up to a constant factor, to the partition function at imaginary chemical potential, i.e., $\tilde{\rho}_{k} \propto Z_{c}(T, k)$, and $\bar{\rho}(\theta) \propto \mathcal{Z}^{I}(\theta)$ or $\rho(s) \propto \mathcal{Z}(i s)$.

Moreover, one easily verifies that the truncated coefficients $\tilde{\rho}_{k}$ at finite $K$ obtained from the discrete Fourier transform in (21), then yield pseudocanonical partition functions, $\tilde{\rho}_{k} \propto Z_{c}^{K}(T, k)$, which represent ensembles with particle number $N=k \bmod K$. Likewise, the discrete sampling of $\bar{\rho}(\theta)$ provides us with an interpolation of $\mathcal{Z}^{I}(\theta)$ which agrees with the exact result for imaginary chemical potential at the discrete values $\theta_{n}=2 \pi n / K$.

The general relation between $\rho(s)$ and the partition function at imaginary chemical potential of course also follows from Eq. (22), with $\mu=i s$ (and $K \rightarrow \infty$ ),

$$
\begin{aligned}
\mathcal{Z}(i s) & =\sqrt{\frac{\pi U}{N_{c} T}} \sum_{k=-\infty}^{\infty} \tilde{\rho}_{k} \exp \left\{-\frac{U}{4 N_{c} T} k^{2}\right\} e^{-i s k / T} \\
& \rightarrow \sqrt{\frac{\pi U T}{N_{c}}} \rho(s), \quad \frac{U}{N_{c} T} \rightarrow 0 .
\end{aligned}
$$

In a finite volume, on the other hand, i.e., at any finite number $N_{c}$ of unit cells, the particle numbers $N$ are restricted to values between $\pm 2 N_{c}$, with $N=0$ at half filling, corresponding to an average of one of the maximally possible two electrons on each of the $2 N_{c}$ sites. We then obtain the exact canonical partition functions from Eq. (23) already for

$$
K=K_{\max }=4 N_{c}+1,
$$

and with particle-hole symmetry at half filling, one actually only needs $K_{\max }=2 N_{c}+1$.

In principle, the particle number $N(\mu)$ can be directly obtained from Eq. (22), which is free of oscillating terms, by taking the derivative with respect to $\mu$,

$$
\begin{aligned}
N(\mu) & =T \frac{d}{d \mu} \ln \mathcal{Z}(\mu) \\
& =-\frac{\sum_{k=0}^{4 N_{c}} k \tilde{\rho}_{k} \exp \left\{-\frac{U}{4 N_{c} T} k^{2}-\frac{\mu}{T} k\right\}}{\sum_{k=0}^{4 N_{c}} \tilde{\rho}_{k} \exp \left\{-\frac{U}{4 N_{c} T} k^{2}-\frac{\mu}{T} k\right\}} .
\end{aligned}
$$

Computing the $\tilde{\rho}_{k}$ from $\rho\left(s_{n}\right)$ can be done with high numerical precision using modern FFT libraries.

Alternatively, we can also compute the chemical potential from the canonical partition functions, as the free energy difference of ensembles with subsequent particle numbers. From Eq. (22) we then obtain

$$
\begin{aligned}
\mu(N+1 / 2) & =-T\left(\ln Z_{c}(T, N+1)-\ln Z_{c}(T, N)\right) \\
& \approx T\left[\ln \tilde{\rho}_{N}-\ln \tilde{\rho}_{N+1}+\frac{U}{2 N_{c} T}(N+1 / 2)\right],
\end{aligned}
$$


and obtain the density in the form of the number of particles per unit cell, $n(\mu) \equiv N(\mu) / N_{c}$, by inversion. The exact calculation would again require $K_{\max }=2 N_{c}+1$ Fourier coefficients. This is then similar in spirit to Refs. [38-40], which carried out canonical calculations of QCD at finite charge density, or Ref. [41] which followed essentially the same strategy for finite isospin density from the lowest states in multipion correlators. With truncating at $K<K_{\max }$, we strictly speaking obtain canonical ensembles at particle number $N$ modulo $K$ as discussed above. The term $\propto U / 2 N_{c} T$ in Eq. (26) represents an explicit finite-volume effect which, as we will discuss below, only contributes in trivial way and can be dropped.

In tight-binding or mean-field calculations, there is no such term in the first place, and the generalized DOS can be calculated analytically. The result is of the form

$\ln \rho(s)=2 N_{c} \int d \varepsilon \rho_{\varepsilon}(\varepsilon) \ln \left(\cosh ^{2} \frac{\varepsilon}{2 T}-\sin ^{2} \frac{s}{2 T}\right)$,

where $\varepsilon \geq 0$ is the single-particle energy with spectral density $\rho_{\varepsilon}(\varepsilon)$ for which an analytic expression is known in the infinite system [42]. In a finite system with periodic (Born-von Kármán) boundary conditions we use the dispersion relation $\varepsilon=\varepsilon(\mathbf{k})$ instead, and simply sum over the corresponding discrete set of points $\mathbf{k}_{n}$ within the first Brillouin zone, with energies $\varepsilon_{n}=\varepsilon\left(\mathbf{k}_{n}\right)$. The same can be done to compute the exact density in the finite system with $N_{c}$ unit cells which then yields for the number of particles per unit cell,

$$
n(\mu)=\frac{1}{N_{c}} \sum_{n}\left(\tanh \frac{\varepsilon_{n}+\mu}{2 T}-\tanh \frac{\varepsilon_{n}-\mu}{2 T}\right) .
$$

We have carried out a set of benchmark calculations in which we compared the canonical and grand-canonical reconstruction schemes. Thereby, a discrete set of values $l_{n}=\ln \rho\left(s_{n}\right)$ for $s_{n}=2 \pi T n / K, N=\{0, \ldots K-1\}$ was produced as mock data from the tight-binding calculation, which can efficiently be done with arbitrary numerical precision. High-precision calculations are especially important in the reconstruction of the density because we need with high precision the discrete Fourier transform of $\left\{\rho_{n}=\right.$ $\left.e^{l_{n}}\right\}$ rather than that of $\left\{l_{n}\right\}$. The number density $n(\mu)$ was subsequently computed from the FFT result $\left\{\tilde{\rho}_{k}\right\}$, using both the fugacity expansion via (25) and the canonical approach (26). We have then compared both results with the exact calculation of the density based on the tightbinding formula (28). This was done for different setups, whereby the production of $\left\{l_{n}=\ln \rho\left(s_{n}\right)\right\}$ was done with different levels of floating point precision. The application of the reconstruction scheme was done with a 1024 digit accuracy in each case to avoid additional errors. We find that both methods yield comparable results, with the canonical procedure having a very slight advantage for a

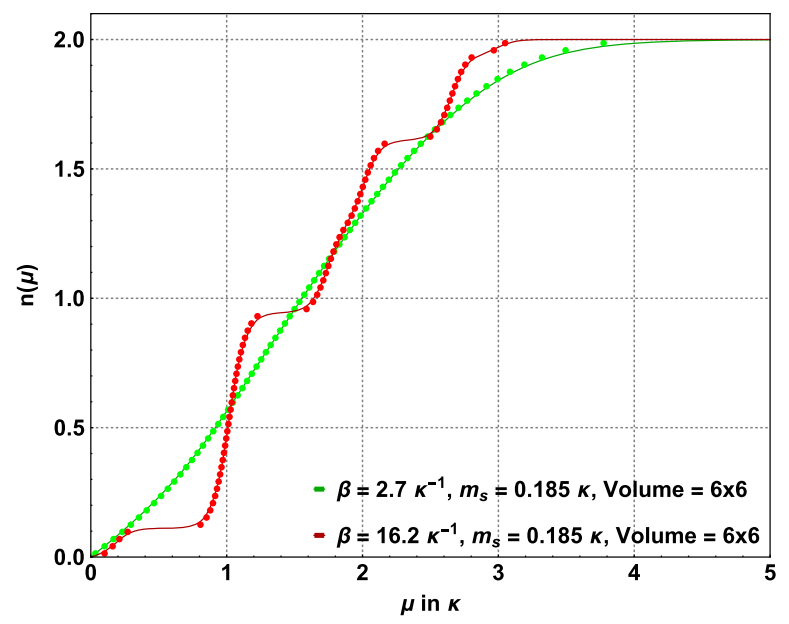

FIG. 2. The number of particles $n(\mu)$ per unit cell on a lattice with $N_{c}=36$ unit cells for two different temperatures at $U=0$ from the tight-binding calculation (solid lines) and from the canonical reconstruction procedure based on (26) using input data of 1024 digit accuracy (discrete points).

given precision of $\ln \rho(s)$. We thus choose to use this procedure exclusively in the following sections to process our LLR results.

Figure 2 shows an example calculation of $n(\mu)$ for two different temperatures on a lattice with $N_{c}=36$ unit cells, where $\ln \rho(s)$ was produced for $U=0$ with 1024 digit accuracy and processed using (26). This illustrates that our method can in principle cover the entire width of the valence band, from the empty valence band at half filling up to saturation when it is completely filled. The van Hove singularity will emerge at $\mu=\kappa$ in the infinite-volume limit which can here be anticipated already by the rapid increase in the number density at the lower temperature around $\mu=\kappa$.

In practice, the leading source of errors is of course the precision with which the Fourier coefficients $\tilde{\rho}_{k}$ can be obtained, which in turn is highly sensitive to statistical errors of $\ln \rho(s)$ in our LLR calculations. In order to get to saturation, with a completely filled lattice, we would obviously need the maximum number $K_{\max }=2 N_{c}+1$ of coefficients. So the double challenge here will be to compute as many of them as accurately as possible.

\section{LLR RESULTS}

\section{A. The algorithm}

The goal of LLR is to calculate derivatives of $\ln \rho(s)$ at a sufficiently dense set of supporting points with high precision and to reconstruct $\rho(s)$ by integration. We divide the domain of support of $\rho(s)$ into $K$ intervals of size $\delta_{s}$. At the center of each of these intervals, the slope $a_{k}=\left.\frac{d}{d s} \ln \rho(s)\right|_{s=s_{k}}$ can be calculated from a stochastic nonlinear equation [5]. A key element of this 
equation is the restricted and reweighted expectation value $^{3}$

$$
\begin{aligned}
\langle\langle W(\Phi)(a)\rangle\rangle_{k}= & \frac{1}{\mathcal{Z}_{\mathrm{LLR}}} \int \mathcal{D} \phi \theta_{\left[s_{k}, \delta_{s}\right]}(\Phi)|\operatorname{det} M(\phi)|^{2} \\
& \times W(\Phi) e^{-\beta S(\phi)} e^{-a \Phi} .
\end{aligned}
$$

Here $\mathcal{Z}_{\mathrm{LLR}}$ is a normalization constant, $\Phi$ was introduced in Eq. (15), $a$ is an external parameter and $\theta_{\left[s_{k}, \delta_{s}\right]}$ is a window function which restricts $\Phi$ to an interval of size $\delta_{s}$ around $s_{k}$.

With the choice $W(\Phi)=\Phi-s_{k}$, the coefficients $a_{k}$ are solutions of

$$
\left.\left\langle W(\Phi)\left(a_{k}\right)\right\rangle\right\rangle_{k}=0
$$

This equation can be solved through Robbins-Monro iteration [43]: The sequence

$$
a_{k}^{(n+1)}=a_{k}^{(n)}+\frac{\alpha_{n}}{\delta_{s}^{2}}\left\langle\left\langle W(\Phi)\left(a_{k}^{(n)}\right)\right\rangle_{k}\right.
$$

converges to the correct result for any choice of $\alpha_{n}$ that fulfills

$$
\sum_{n=0}^{\infty} \alpha_{n}=\infty, \quad \sum_{n=0}^{\infty} \alpha_{n}^{2}<\infty .
$$

This is true, even if $\langle\langle W(\Phi)(\cdot)\rangle\rangle_{k}$ is approximated by an estimator, as we do in Monte Carlo calculations. Moreover, if the iteration is terminated at some finite number $N$ and repeated many times, the final values $a_{k}^{(N)}$ are Gaussian distributed around the true value $a_{k}$ and can be processed by a standard bootstrap analysis.

The window function can be chosen in different ways. The straightforward choice is a step function, but for Hybrid Monte Carlo (HMC) a Gaussian window function is more appropriate, as its derivative can be taken, which implies that its effect can be reproduced by a moleculardynamics force term. In this work, we choose

$$
\begin{aligned}
\langle\langle\Phi-s\rangle\rangle(a) & \\
= & \frac{1}{\mathcal{Z}_{\mathrm{LLR}}} \int \mathcal{D} \phi \operatorname{det} M(\phi) \operatorname{det} M^{\dagger}(\phi)(\Phi-s) \\
& \times \exp \left\{-\frac{\delta}{2 U} \sum_{x, t}\left(\phi_{x, t}-s\right)^{2}-\frac{1}{2 \delta_{s}^{2}}(s-\Phi)^{2}-a \Phi\right\},
\end{aligned}
$$

where

\footnotetext{
${ }^{3}$ The double-bracket notation is customary in the LLR literature and should be understood as defined by Eq. (29). It is not implied here that an expectation value is taken twice.
}

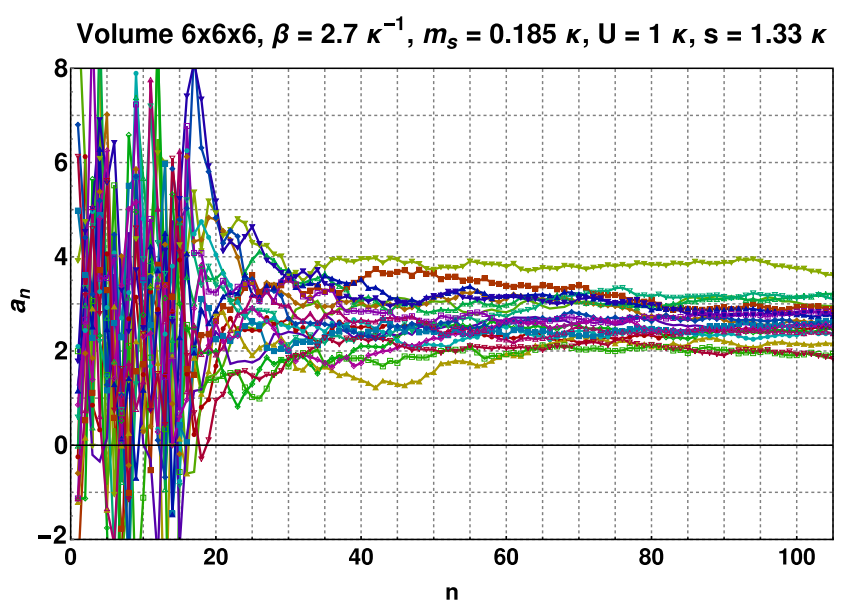

FIG. 3. Illustration of stochastic Robbins-Monro iteration. A set of 20 starting values $a_{k}^{(0)}$ are generated and are each updated according to Eq. (31). Under-relaxation is switched on at $n=15$. The procedure is terminated at $n=105$ to obtain the final values used for bootstrapping.

$$
\begin{aligned}
& \mathcal{Z}_{\mathrm{LLR}}(a) \\
& =\int \mathcal{D} \phi \operatorname{det} M(\phi) \operatorname{det} M^{\dagger}(\phi) \exp \left\{-\frac{\delta}{2 U} \sum_{x, t}\left(\phi_{x, t}-s\right)^{2}\right. \\
& \left.\quad-\frac{1}{2 \delta_{s}^{2}}(s-\Phi)^{2}-a \Phi\right\}
\end{aligned}
$$

The full procedure is then summarized as follows:

(1) For a given $s_{k}$, initialize $a_{k}$ with some random value $a_{k}^{(0)}$ not too far from zero.

(2) Initialize Hubbard field (e.g., with a value which minimizes the window function).

(3) With fixed $a_{k}$, thermalize Hubbard field with HMC trajectories according to Eq. (34), i.e., $\mathcal{Z}_{\mathrm{LLR}}\left(a_{k}\right)$.

(4) With additional HMC trajectories, compute an estimate of $\langle\langle\Phi-s\rangle\rangle\left(a_{k}\right)$.

(5) Update $a_{k}$ using Eq. (31).

(6) Continue from step 3.

In practice, we start with several repetitions of steps 3-6 with fixed $\alpha=1$ and switch to under-relaxed interations with $\alpha_{n+1}=\alpha_{n} /(1+n)$ after some time. Also, the whole procedure is terminated after some finite iteration number $N$ and repeated several times for each fixed $s_{k}$, to produce a sample of final $a_{k}^{(N)}$ values.

Figure 3 shows one example of a stochastic RobbinsMonro iteration taken from our actual production runs, where the procedure described above is applied for a fixed set of external parameters. We choose $N_{s}=N_{t}=6, \beta=$ $2.7 \kappa^{-1}, m_{s}=0.185 \kappa, U=1.0 \kappa, s=1.33 \kappa$ for illustration. ${ }^{4}$

\footnotetext{
${ }^{4}$ Note that the phenomenological value of the hopping parameter in the tight-binding model for graphene typically is $\kappa \approx 2.7 \mathrm{eV}$, so this would correspond to a temperature of $T \approx 1 \mathrm{eV}$ in graphene.
} 

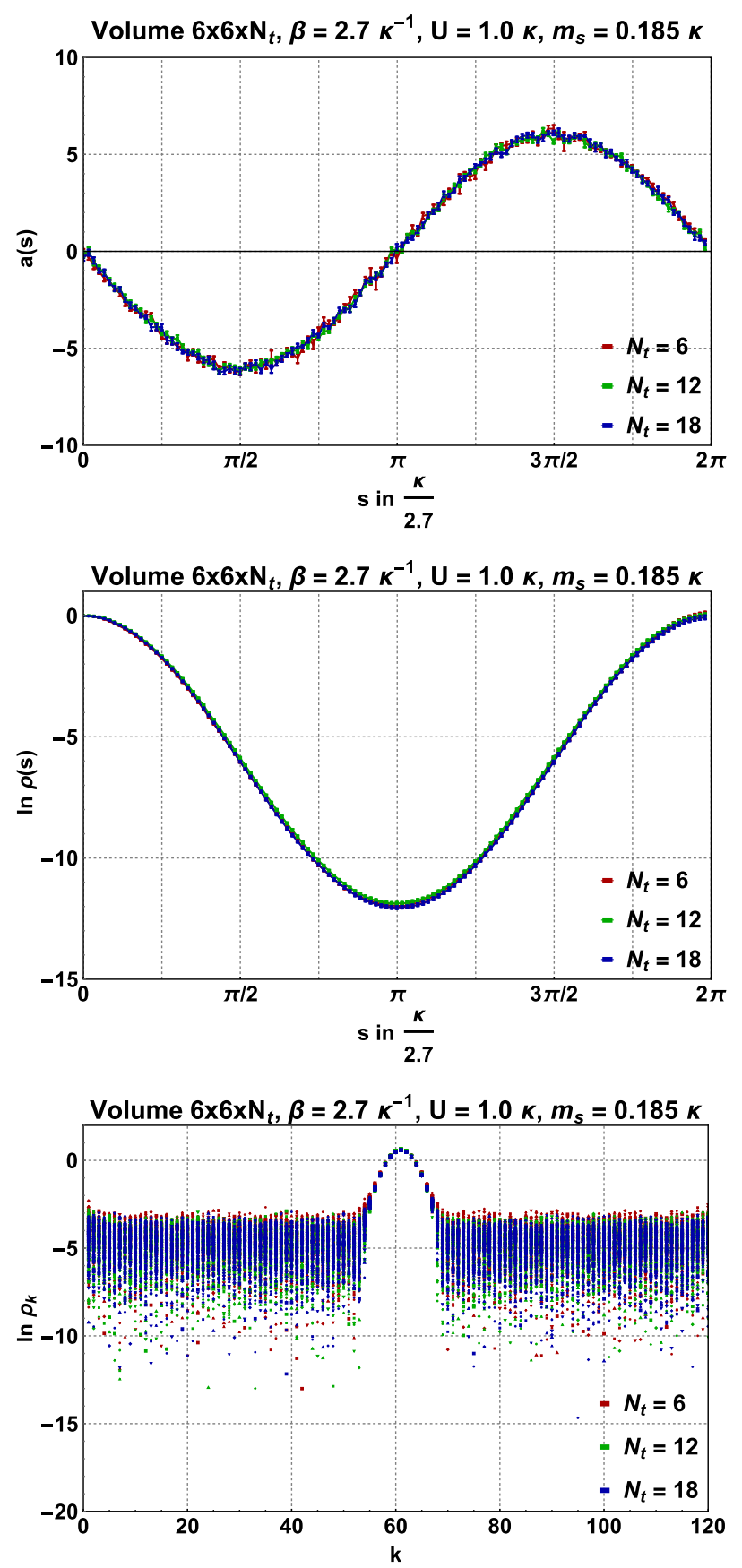

FIG. 4. LLR result: $N_{t}$ dependence of $a(s), \ln \rho(s), \ln \tilde{\rho}_{k}$ for $N_{s}=6, \beta=2.7 \kappa^{-1}, U=1.0 \kappa, m_{s}=0.185 \kappa$. Individual bootstrap averages are shown for $\ln \tilde{\rho}_{k}$ to illustrate loss of signal for the higher modes.

For each set of parameters considered in this work, we first obtain such a sample of $a_{k}$ values. We then obtain $\ln \rho\left(s_{k}\right)$, and by extension $\rho\left(s_{k}\right), \tilde{\rho}_{k}$ and $n(\mu)$ together with error bars, by feeding bootstrap averages of the final $a_{k}^{(N)}$ into

$$
\ln \rho\left(s_{k}\right)=\sum_{i=0}^{k-1} a_{i} \delta_{s}+\frac{1}{2} a_{k} \delta_{s}
$$
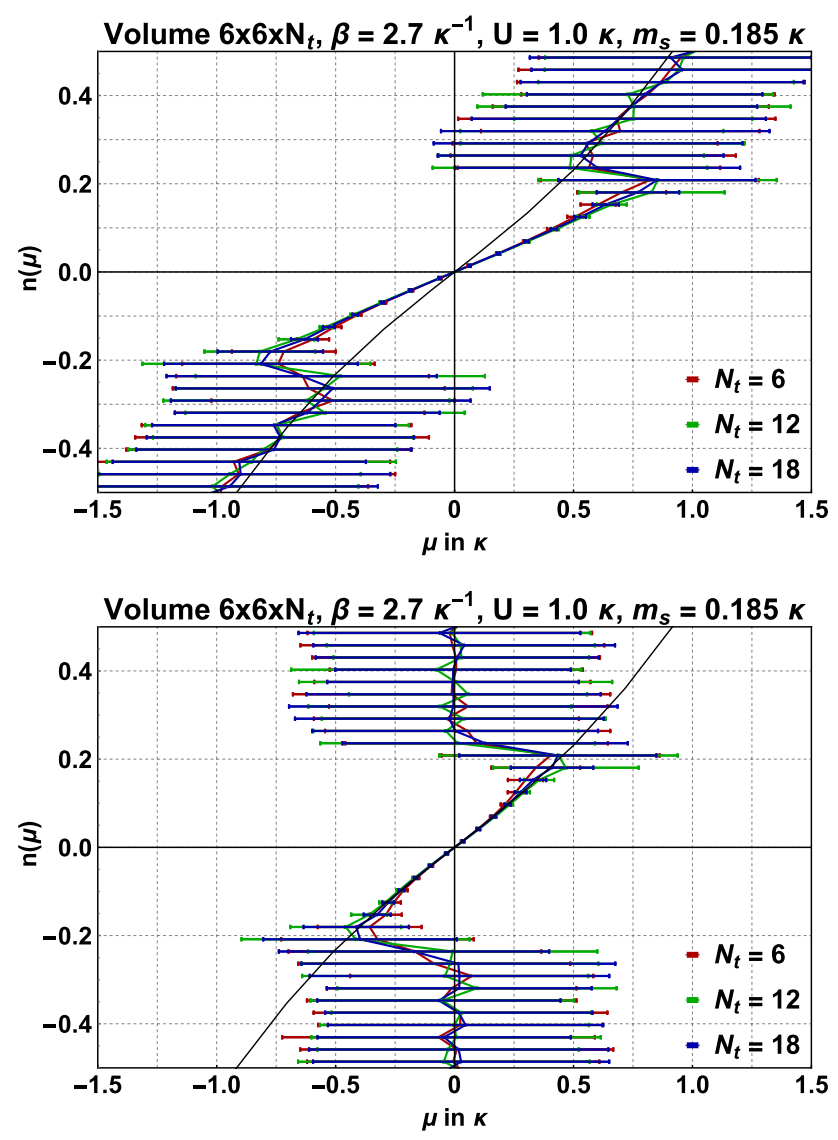

FIG. 5. LLR result: $N_{t}$ dependence of $n(\mu)$ for $N_{s}=6$, $\beta=2.7 \kappa^{-1}, U=1.0 \kappa, m_{s}=0.185 \kappa$. Top figure includes the linear $(\sim U)$ term in Eq. (26), bottom figure does not. Error bars computed by bootstrapping. Solid line shows the noninteracting tight-binding theory.

computing the Fourier transform of $\rho\left(s_{k}\right)$ and applying the canonical reconstruction scheme described in Sec. IV.

\section{B. $N_{t}$ dependence}

We begin by studying the effect of the time discretization $\delta$. To this end, we carry out LLR calculations at $N_{s}=6$, $\beta=2.7 \kappa^{-1}, U=1.0 \kappa, m_{s}=0.185 \kappa$ for different values of $N_{t}$. Figure 4 shows the results for $a(s), \ln \rho\left(s_{k}\right)$ and $\ln \tilde{\rho}_{k}$, while the final results for $n(\mu)$ are shown in Fig. 5. The latter figure includes two subfigures, whereby the linear $(\sim U)$ contribution to Eq. (26) is included or neglected respectively. Figure 5 also shows a corresponding calculation of $n(\mu)$ in the noninteracting tight-binding theory. All error bars were obtained through bootstrap analysis.

Our first observation is that the dependence on $N_{t}$ is very mild for our choice of parameters. It is practically invisible in $a(s)$ and $n(\mu)$. A very small difference between different $N_{t}$ can be seen in $\ln \rho\left(s_{k}\right)$ and $\ln \tilde{\rho}_{k}$, which is of a similar magnitude as the statistical uncertainty however. On the other hand, our results clearly demonstrate exponential error suppression, whereby the relative error of $\ln \rho(s)$ is 
roughly the same across several orders of magnitude. We find that $\ln \tilde{\rho}_{k}$ is extremely sensitive to this small error however, to a degree that only the first few Fourier modes $\ln \tilde{\rho}_{k}$ can be computed accurately. This can be traced back to the fact that $\rho(s)$ enters into the Fourier transform and not $\ln \rho(s)$. It is also reflected in our computation of $n(\mu)$, which exhibits a loss of signal at $\mu \approx 0.5 \kappa$, indicating the onset of a hard sign problem.

We note that for $U=1.0 \kappa$ which is well inside the weakcoupling phase of the model, and the temperature considered here, $n(\mu)$ basically fully agrees with the infinitevolume limit in the noninteracting theory when the linear term in Eq. (26) is dropped. We take this as an indication that this extra term represents the dominant finite-volume effect at finite $U$ which however is a rather trivial one to correct. Further confirmation of this is provided by a comparison between results from $N_{s}=6$ and $N_{s}=12$ lattices, which also reveals a faster convergence to the thermodynamic limit without this term. We thus drop this term for all results presented in the following. We expect that deviations from the noninteracting limit will become visible at stronger couplings, of course. This is investigated further, and ultimately confirmed, below.

\section{C. $m_{s}$ dependence}

We now turn to studying the dependence on the explicit sublattice and spin-staggered mass term $m_{s}$. Given that such a term already opens an explicit gap in the energy spectrum, we carry out this study at the comparatively weak coupling strength of $U=0.1 \kappa$. We find that, again, the number density $n(\mu)$ coincides with the noninteracting theory and shows no significant dependence on $m_{s}$. The linear term in Eq. (26) has a negligible effect here, due to the small value of $U$. Figure 6 shows the results for $a(s)$ and $\ln \rho(s)$ with $N_{s}=N_{t}=6, \beta=2.7 \kappa^{-1}$ and three different choices of $m_{s}$. We refrain from showing any additional figures for $\ln \tilde{\rho}_{k}$ and $n(\mu)$, as these fully agree (within statistical errors) with the results shown in the lowest panels of Figs. 4 and 5.

An interesting observation here is that $m_{s}$ has a quite strong effect on both $a(s)$ and $\ln \rho(s)$, which turns out not to carry over to $n(\mu)$ at all. The underlying reason is that this dependence is only present in regions where $\rho(s)$ is strongly suppressed. It is only visible due to the logarithmic scale, and thus has no significant effect on the computation of the Fourier modes.

\section{D. $U$ dependence}

Having validated our numerical procedure at weak coupling, we now turn to a more detailed study of the dependence on the interaction strength $U$. This represents the central part of this work to which the bulk of our computing resources were dedicated. We thereby computed $a(s), \ln \rho(s), \ln \tilde{\rho}_{k}$ and $n(\mu)$ again with $\beta=2.7 \kappa^{-1}$,
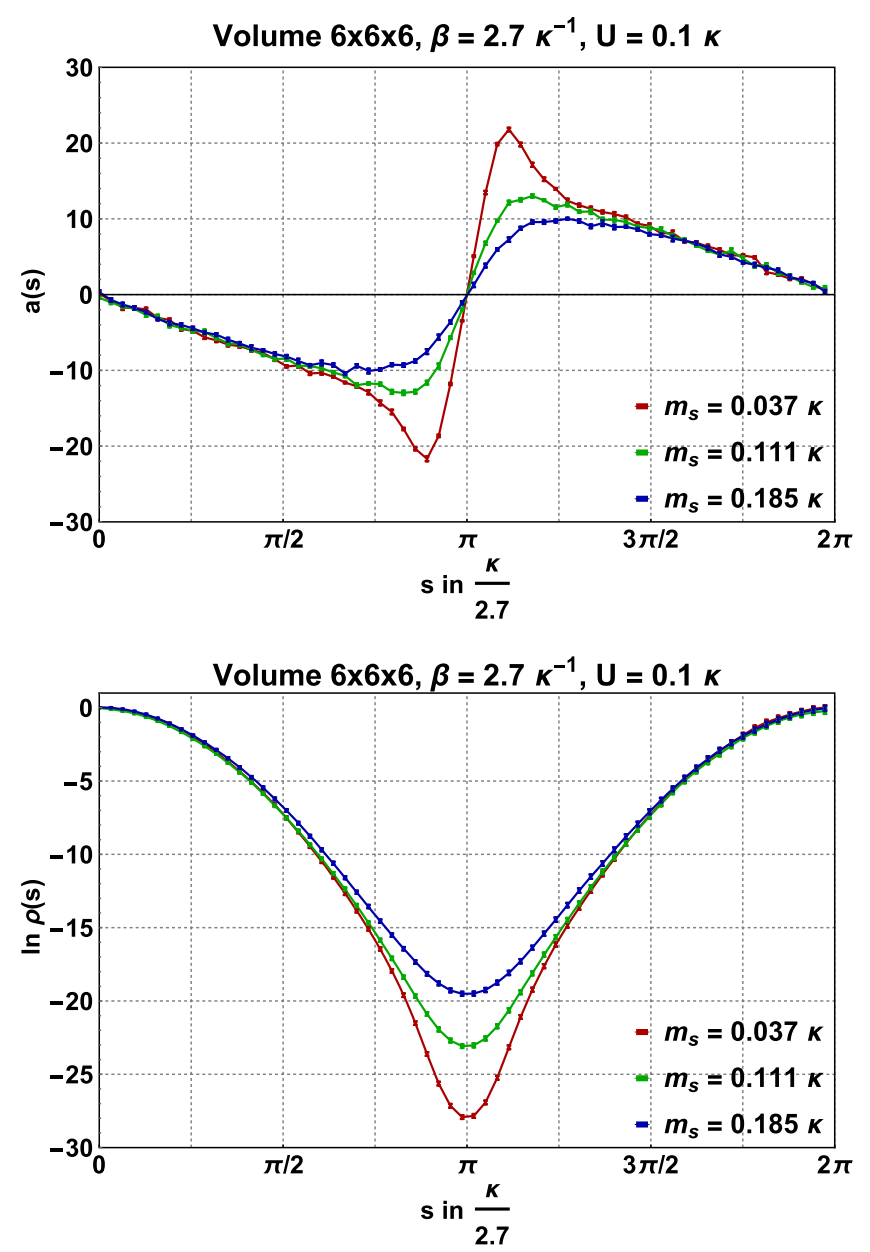

FIG. 6. LLR result: $m_{s}$ dependence of $a(s)$ and $\ln \rho(s)$ for $N_{s}=N_{t}=6, \beta=2.7 \kappa^{-1}, U=0.1 \kappa$.

$m_{s}=0.185 \kappa$ for several different choices of $U$. To have control over finite-volume and time-discretization effects we have studied two different lattice sizes, $N_{s}=N_{t}=6$ and $N_{s}=N_{t}=12$, respectively.

Figure 7 shows the $U$ dependence of $a(s), \ln \rho(s)$ and $\ln \tilde{\rho}_{k}$ for $N_{s}=N_{t}=6, \beta=2.7 \kappa^{-1}, m_{s}=0.185 \kappa$. For $\ln \rho(s)$ we include the tight-binding result to illustrate the approach to the noninteracting limit. The first observation is that $a(s)$ gets suppressed when $U$ is increased, which ultimately makes simulations more expensive at strong coupling. On the other hand, we clearly see a deviation from the noninteracting limit in the Fourier modes $\ln \tilde{\rho}_{k}$ for the strongest interaction strength $U=2.0 \kappa$. To underscore that this deviation is absent for all weaker interactions, we show a separate plot in Fig. 8 which directly compares $\ln \tilde{\rho}_{k}$ for $U \leq 1.0 \kappa$ to the tight-binding theory. Our $N_{s}=N_{t}=6$ results for $n(\mu)$ are shown in Fig. 9. They clearly show a corresponding drop of the number density at fixed $\mu$ for the strongest coupling.

$N_{s}=12$ results are shown in Fig. 10 for $a(s), \ln \rho(s)$ and $\ln \tilde{\rho}_{k}$ and Fig. 11 for $n(\mu)$. These confirm the qualitative changes at $U=2.0 \kappa$. Furthermore, a direct 

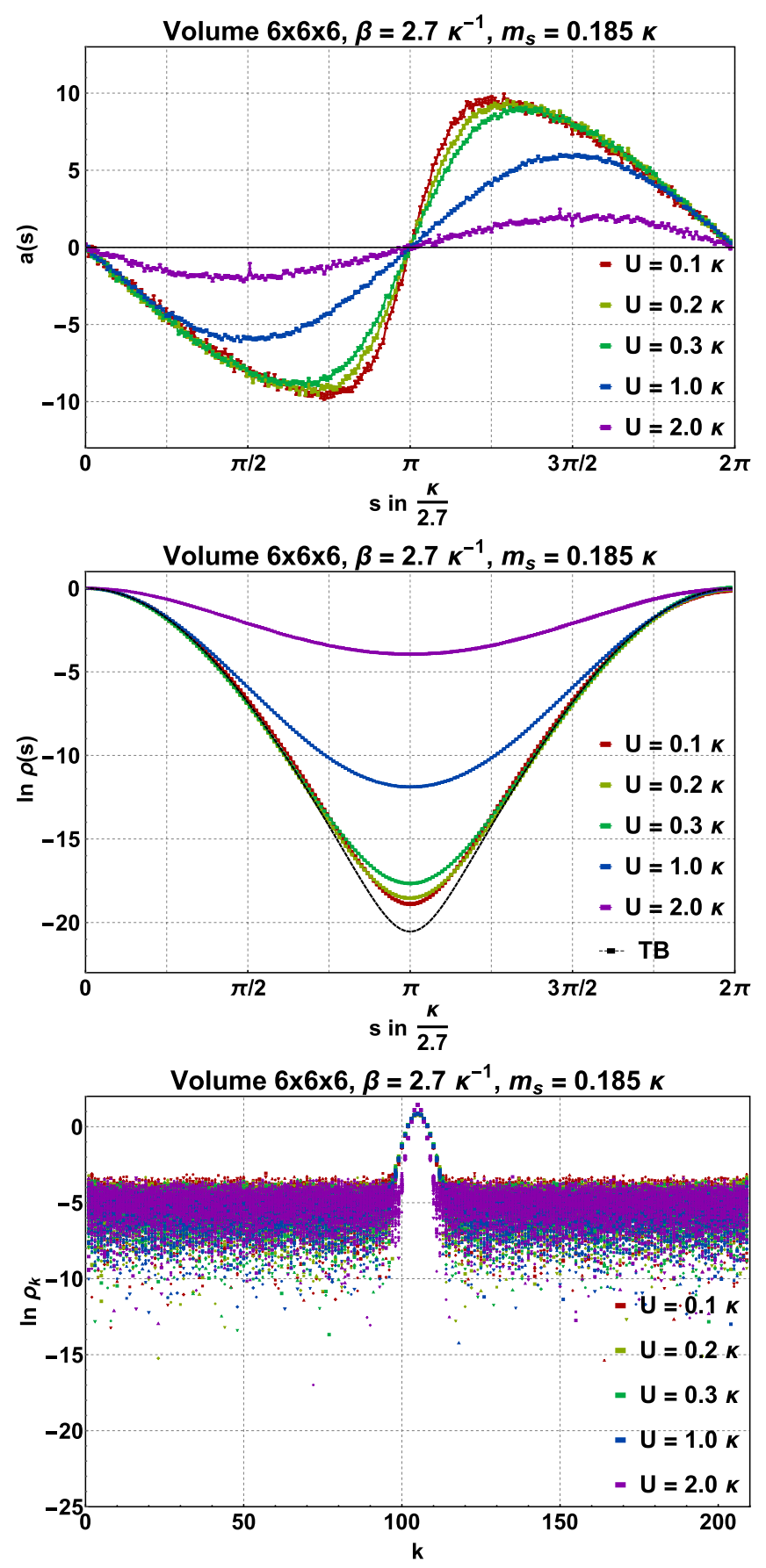

FIG. 7. LLR result: $U$ dependence of $a(s), \ln \rho(s), \ln \tilde{\rho}_{k}$ for $N_{s}=N_{t}=6, \beta=2.7 \kappa^{-1}, m_{s}=0.185 \kappa$. Individual bootstrap averages are shown for $\ln \tilde{\rho}_{k}$. Result for noninteracting tightbinding theory is included for $\ln \rho(s)$.

comparison with $N_{s}=6$ suggests that finite-volume effects on $n(\mu)$ are rather mild.

We point out here that the sign problem sets in at much smaller $\mu$ for the larger system (as expected). While we are able to reliably compute $n(\mu)$ up to $\mu \approx 0.35 \kappa$ for $N_{s}=6$ with $U=1.0 \kappa$, we only reach $\mu \approx 0.1 \kappa$ for $N_{s}=12$. On the other hand, in both cases LLR drastically outperforms

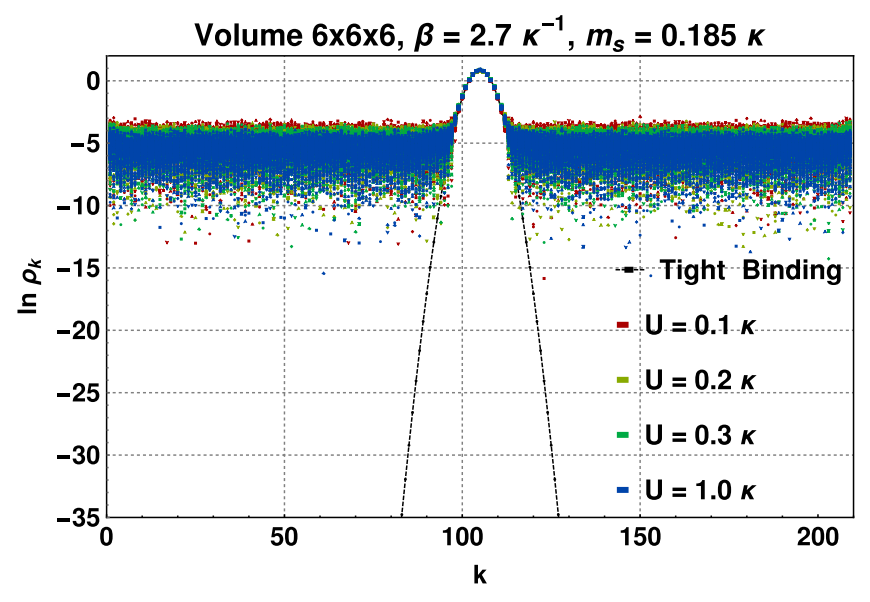

FIG. 8. LLR result: $\ln \tilde{\rho}_{k}$ for $N_{s}=N_{t}=6, \beta=2.7 \kappa^{-1}, m_{s}=$ $0.185 \kappa$ and different $U$ compared with noninteracting tightbinding theory.

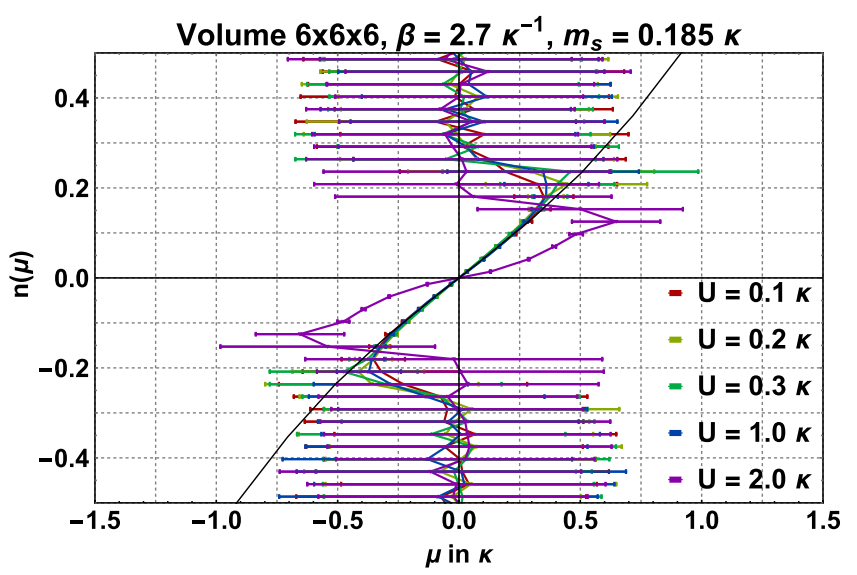

FIG. 9. LLR result: $U$ dependence of $n(\mu)$ for $N_{s}=N_{t}=6$, $\beta=2.7 \kappa^{-1}, m_{s}=0.185 \kappa$. Error bars computed by bootstrapping. Solid line shows the noninteracting tight-binding theory.

brute-force reweighting: With comparable numerical resources we obtain a signal for the determinant ratio (8) up to $\mu \approx 0.14 \kappa$ on $N_{s}=6$ and $\mu \approx 0.075 \kappa$ on $N_{s}=12$ using the brute-force method. While the relative advantage of LLR becomes smaller on the larger lattice, we can reach much larger values of $\mu$ for $U=2.0 \kappa\left(\mu \approx 0.5 \kappa\right.$ on $N_{s}=6$ and $\mu \approx 0.2 \kappa$ on $N_{s}=12$ ). In contrast, the $\mu$ range of reweighting is drastically diminished at stronger coupling (cf. Fig. 13 in Sec. VI). It is this last feature which ultimately makes LLR in its present form a promising method and deserving of further attention.

\section{E. Compressed sensing}

Lastly, we report on our attempts to improve our results by using fit functions for $\ln \rho(s)$, a procedure referred to as compressed sensing in the LLR literature. The basic idea is to, instead of processing the raw data for $\ln \rho(s)$ pointwise 

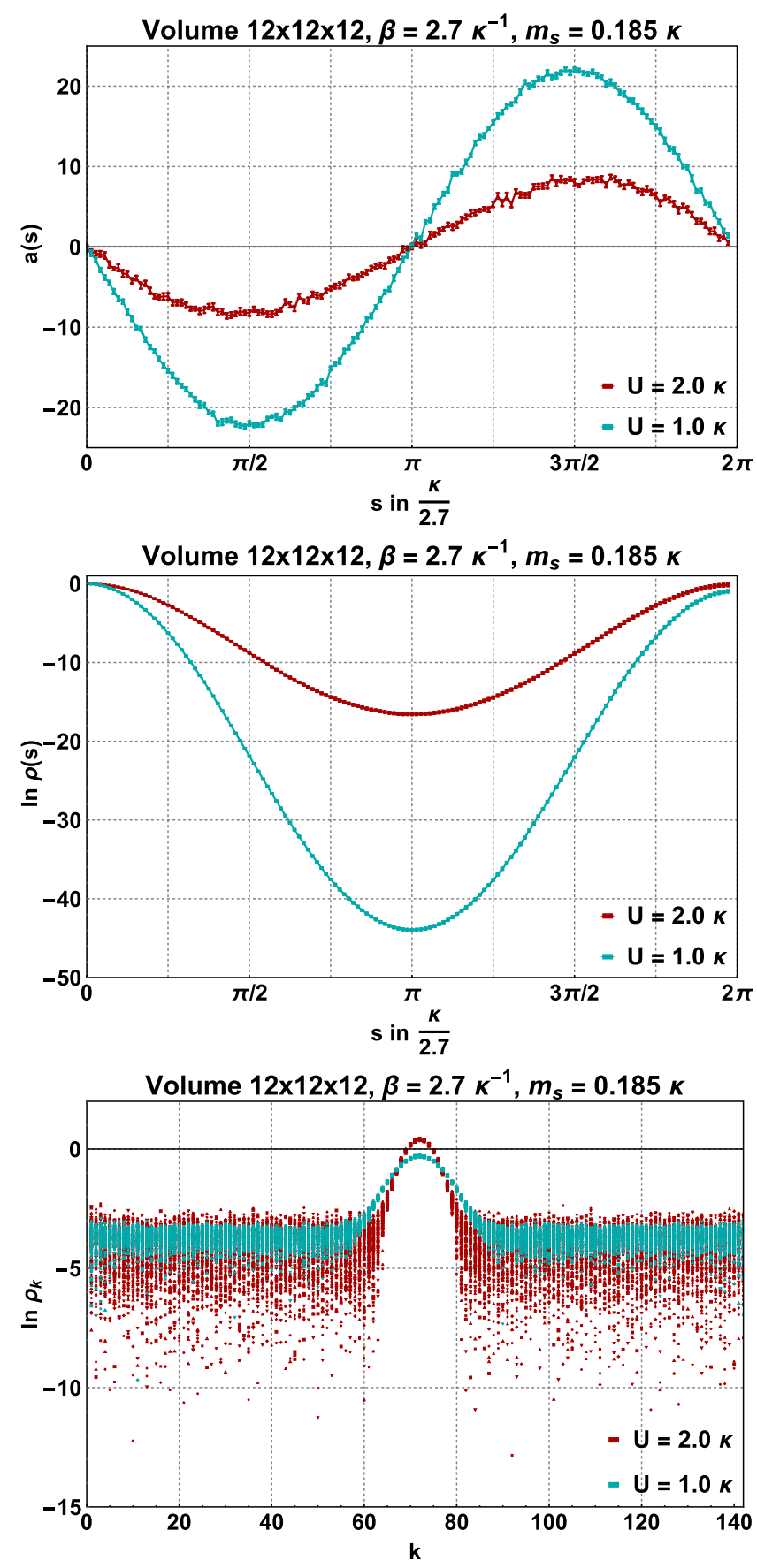

FIG. 10. LLR result: $U$ dependence of $a(s), \ln \rho(s), \ln \tilde{\rho}_{k}$ for $N_{s}=N_{t}=12, \beta=2.7 \kappa^{-1}, m_{s}=0.185 \kappa$. Individual bootstrap averages are shown for $\ln \tilde{\rho}_{k}$.

at the supporting points $s_{k}$, fit the entire dataset with a series expansion in some complete set of functions, and use the model curve to compute observables instead. The hope is that an appropriate set of functions, which reflects the true (but a priori unknown) physics of the theory, will both suppress noise in the numerical data for $\ln \rho(s)$ and effectively generate an interpolation to a much denser set of supporting points. This in turn should allow for

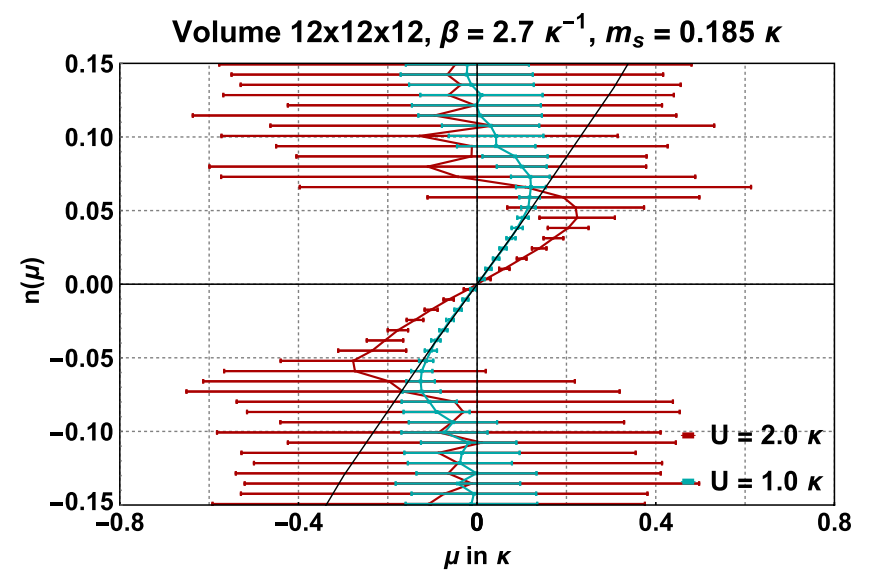

FIG. 11. LLR result: $U$ dependence of $n(\mu)$ for $N_{s}=N_{t}=12$, $\beta=2.7 \kappa^{-1}, m_{s}=0.185 \kappa$. Error bars computed by bootstrapping. Solid line shows the noninteracting tight-binding theory.
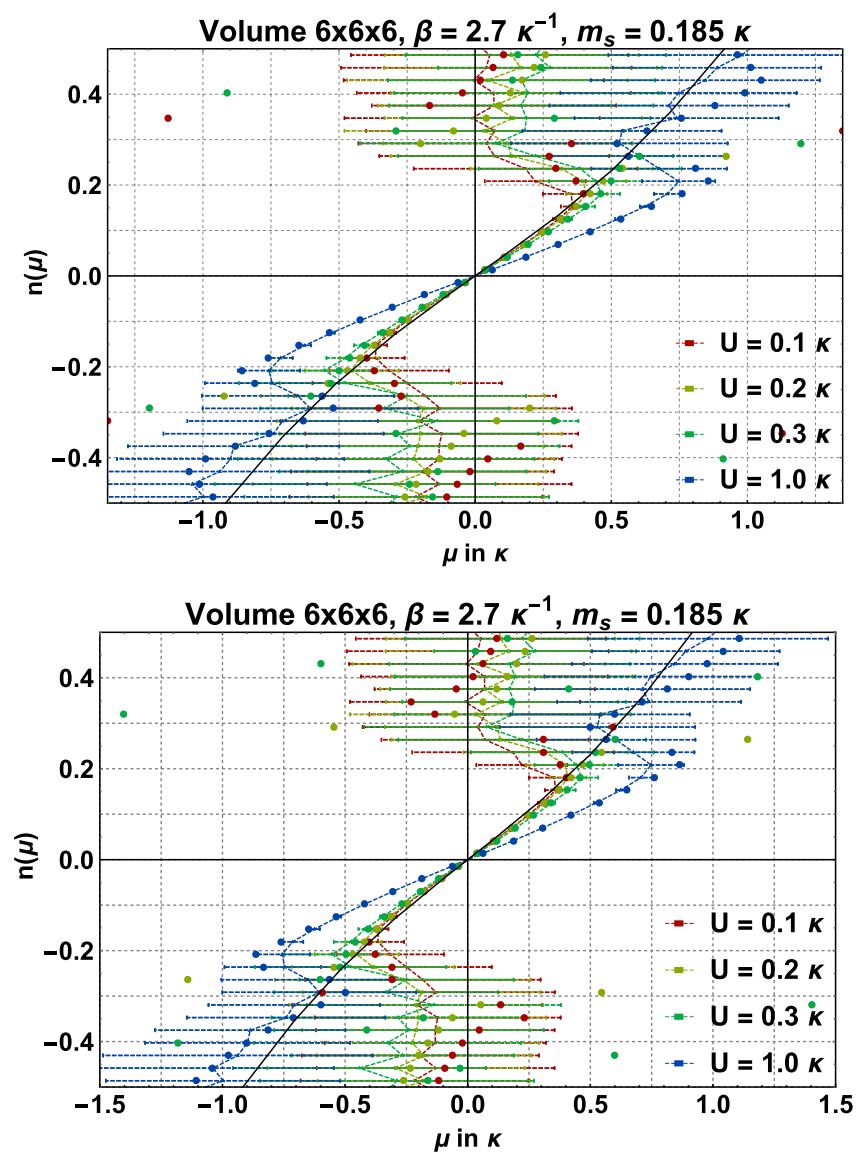

FIG. 12. LLR result: $\mu$ dependence of particle density for $N_{s}=N_{t}=6, \beta=2.7 \kappa^{-1}, m_{s}=0.185 \kappa$ and different $U$ [results include the linear $\sim U$ term in Eq. (26)]. Dashed lines were obtained directly from $\ln \tilde{\rho}_{k}$, while dots employed compressed sensing: $\ln \rho(s)$ was fit with a Fourier series (top figure) and Chebyshev polynomials (bottom figure) respectively. Solid line shows noninteracting tight-binding theory. 


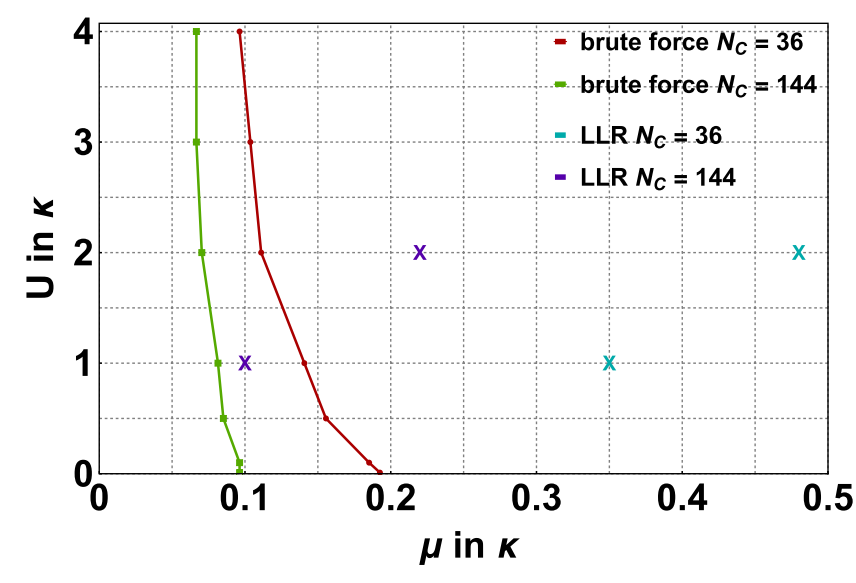

FIG. 13. Comparison of effective $\mu$ range of brute-force reweighting and LLR (results shown for $6^{3}$ and $12^{3}$ lattices at $\left.\beta=2.7 \kappa^{-1}\right)$. For each value of $U$ the phase distribution of $\operatorname{det} \tilde{M}(\phi, \mu) / \operatorname{det} \tilde{M}(\phi,-\mu)$ was measured at different $\mu$ until the signal was lost. A roughly equal amount of computer time was spent for corresponding LLR calculations.

the computation of $\ln \tilde{\rho}_{k}$ at larger $k$ and hence the number density at larger $\mu$.

Figure 12 shows two such attempts, where $\ln \rho(s)$ was fit with a Fourier series and a series of Chebyshev polynomials of the first kind respectively. The fit function was subsequently evaluated at a much denser set of points than the original $s_{k}$ and used to compute $\ln \tilde{\rho}_{k}$ and subsequently $n(\mu)$. In each case, higher-order terms were added to the expansion until the final result stabilized. We do not obtain any error bars. Results from the direct calculation are included for comparison and represented by dashed lines.

We find that these attempts do not improve the calculation of $n(\mu)$ significantly. At best, one or two additional points (at higher densities) can be computed before the results scatter in an uncontrolled fashion. The Fourier series thereby seems to work only slightly better than the Chebyshev polynomials. We take this as an indication that additional qualitative information about the system, which places additional constraints on the choice of functions to use, is a necessary requirement for compressed sensing to be effective here.

\section{CONCLUSION AND OUTLOOK}

In this work, we applied the linear logarithmic relaxation method to the repulsive fermionic Hubbard model on the honeycomb lattice, in order to assess its utility for alleviating the hard sign problem of an unbalanced dynamical fermion system. A central problem thereby is the proper choice of a target observable, which adequately reflects the complex part of the action and yields a generalized density of states which is suitable for further processing. We used the average value $\Phi$ of the auxiliary (Hubbard) field to this end, which appeared as the natural choice, as it allows for the shifting of the complex part of the fermion determinant into the bosonic sector and provides a simple integral expression [Eq. (17)] for obtaining the partition function and hence the particle density. To deal with an oscillating contribution to this integral, we chose to work in the frequency domain and devised two methods to extract the particle density from the Fourier modes of the GDOS of $\Phi$ which essentially yields the partition function at imaginary chemical potential. Due to a slightly better performance in benchmark calculations, of these we chose a method based on the canonical ensembles to further process our LLR results.

We carried out LLR calculations for a fixed temperature of $\beta=2.7 \kappa^{-1}$, two different lattice sizes $\left(6^{3}\right.$ and $\left.12^{3}\right)$ and different interaction strengths in the weak and intermediate coupling regime, and obtained the particle density as a function of chemical potential. We thereby observed significant deviations from the noninteracting theory for the largest interaction strength considered, $U / \kappa=2.0$, signaling strong correlations which might eventually lead to spontaneous mass-gap formation which is known to occur at around $U / \kappa \approx 3.8[12,34]$ in the infinite-volume limit.

We found that using LLR in its present form, on the smaller $6^{3}$ lattice we were able to probe at least twice as far into the finite-density regime as with brute-force reweighting. While the relative advantage of LLR is smaller on the $12^{3}$ lattice, we found that LLR performs much better when the interaction strength is increased. Figure 13 shows a quantitative comparison of the effective $\mu$ ranges for the different parameter sets considered in this work.

Attempts to reach into higher-density regions were made using different forms of compressed sensing, i.e., by fitting $\ln \rho(s)$ with Fourier series and Chebyshev polynomials and using the model curves for interpolation. While this allows us to reach slightly higher densities, we suspect that this procedure introduces an uncontrolled systematic error, as the physics at higher densities is strongly sensitive to the high-frequency modes of $\rho(s)$, which such interpolations cannot account for.

The results presented here should be taken as a proof of principle. There are several different directions for future improvements. First and foremost, the computational resources spent for the final calculations (i.e., the sum of all results shown in Figs. 9 and 11) were around two months of runtime on a total of 18 GTX 980 Ti GPUs, which leaves much space for larger-scale projects. We estimate that, using the most modern hardware and libraries for sparse linear algebra, the precision for $\ln \rho(s)$ can be increased by at least an order of magnitude. More advanced techniques for compressed sensing could also be applied, such as Gaussian and telegraphic approximations or an advanced moments approach, which were proposed in Ref. [44]. Quite possibly, introducing a complex instead of a real auxiliary field has advantages, and in fact, it was shown that an optimal mixing factor between real and imaginary Hubbard fields exists, for which the sign 
problem is the mildest [45]. LLR might also be more effective with a discrete Hubbard field, which is used in Blankenbecler-Scalapino-Sugar Quantum Monte Carlo calculations [46,47]. In addition, an alternative time discretization with a symmetry of time reversal times sublattice exchange, which was proposed already in Ref. [22] and recently used in a grand-canonical HMC simulation [48], was shown to have strongly suppressed discretization effects in Ref. [30] and might positively impact the performance of LLR as well. And finally, there has been much recent progress regarding the Lefschetz thimble method [45,49,50], and constructing a hybrid approach, which combines the advantages of both methods, might be feasible. Specifically, one could attempt to apply the Lefschetz thimble decomposition directly to Eq. (17), in order to avoid the use of reconstruction schemes altogether and obtain a cleaner signal for $n(\mu)$.

Taken together, we find it not unreasonable to expect that future developments might put the van Hove singularity (VHS) of the single-particle spectrum within reach, which is of great interest in the context of superconducting phases. A crucial point thereby is the apparent stability of the LLR technique against increases of the coupling strength $U$. Experiments on charge-doped graphene systems have revealed a strong bandwidth renormalization (narrowing of the width of the $\pi$ bands) due to interactions [20], which suggests that the VHS can be probed at smaller $\mu$ for larger $U$. Furthermore, a HMC study of graphene at finite spin density revealed that the electronic Lifshitz transition at the VHS can become a true thermodynamic phase transition in the presence of interactions, with a critical temperature which increases with the coupling strength [31]. A study of an analogous transition at finite charge-carrier density thus might be feasible at large $U$, in particular as the sign problem becomes milder at higher temperatures.
There are many possibilities to linearize the quartic fermionic interaction using auxiliary fields. The choice in this paper is inspired by the observation that an explicit analytic continuation [i.e., Eq. (12)] was sufficient to split off the complex part of the fermion determinant. The formulation is elegant: The calculation of one (real) density of states, $\rho(s)$, is sufficient to relay the calculation of the partition function to one integration for each given value of the chemical potential. Note, however, that the use of a Hubbard field $\phi$ with a compact domain of support implies that the domain of the density of states is also compact. The calculation of such an "intensive" density of states to sufficient precision is difficult [44] and the most successful LLR calculations for theories with a sign problem are based upon noncompact densities [10]. The use of a noncompact formulation is left to future work.

Lastly, we should mention that extending our work to the QCD sign problem remains an open conceptual challenge. The system considered here was special since we succeeded to remove the complex part the fermion determinant by a simple analytic continuation. For gauge theories no such simple transformation exists, and measuring a proper extensive phase is much more involved. It may well be that this step is the most computationally demanding and contains the central computational complexity of the QCD sign problem.

\section{ACKNOWLEDGMENTS}

This work was supported by the Helmholtz International Center known as HIC for FAIR (Facility for Antiproton and Ion Research) and its successor, the Helmholtz Research Academy Hessen for FAIR. We are grateful to Pavel Buividovich, John Gracey and Maksim Ulybyshev for helpful discussions and comments.
[1] M. Troyer and U.-J. Wiese, Computational Complexity and Fundamental Limitations to Fermionic Quantum Monte Carlo Simulations, Phys. Rev. Lett. 94, 170201 (2005).

[2] A. Bazavov et al., The QCD equation of state to $\mathcal{O}\left(\mu_{B}^{6}\right)$ from lattice QCD, Phys. Rev. D 95, 054504 (2017).

[3] B. A. Berg and T. Neuhaus, Multicanonical Ensemble: A New Approach to Simulate First Order Phase Transitions, Phys. Rev. Lett. 68, 9 (1992).

[4] F. Wang and D. P. Landau, Efficient, Multiple-Range Random Walk Algorithm to Calculate the Density of States, Phys. Rev. Lett. 86, 2050 (2001).

[5] K. Langfeld, B. Lucini, and A. Rago, The Density of States in Gauge Theories, Phys. Rev. Lett. 109, 111601 (2012).
[6] C. Gattringer and K. Langfeld, Approaches to the sign problem in lattice field theory, Int. J. Mod. Phys. A 31, 1643007 (2016).

[7] K. Langfeld, B. Lucini, R. Pellegrini, and A. Rago, An efficient algorithm for numerical computations of continuous densities of states, Eur. Phys. J. C 76, 306 (2016).

[8] K. Langfeld, Density-of-states, Proc. Sci., Lattice2016 (2017) 010 [arXiv:1610.09856].

[9] K. Langfeld and J. M. Pawlowski, Two-color QCD with heavy quarks at finite densities, Phys. Rev. D 88, 071502 (2013).

[10] K. Langfeld and B. Lucini, Density of states approach to dense quantum systems, Phys. Rev. D 90, 094502 (2014).

[11] N. Garron and K. Langfeld, Anatomy of the sign-problem in heavy-dense QCD, Eur. Phys. J. C 76, 569 (2016). 
[12] F. F. Assaad and I. F. Herbut, Pinning the Order: The Nature of Quantum Criticality in the Hubbard Model on Honeycomb Lattice, Phys. Rev. X 3, 031010 (2013).

[13] Y. Otsuka, S. Yunoki, and S. Sorella, Universal Quantum Criticality in the Metal-Insulator Transition of Two-Dimensional Interacting Dirac Electrons, Phys. Rev. X 6, 011029 (2016).

[14] F. Parisen Toldin, M. Hohenadler, F. F. Assaad, and I. F. Herbut, Fermionic quantum criticality in honeycomb and $\pi$-flux Hubbard models: Finite-size scaling of renormalization-group-invariant observables from quantum Monte Carlo, Phys. Rev. B 91, 165108 (2015).

[15] M. Hohenadler, F. Parisen Toldin, I. F. Herbut, and F. F. Assaad, Phase diagram of the Kane-Mele-Coulomb model, Phys. Rev. B 90, 085146 (2014).

[16] J. Lenz, L. Pannullo, M. Wagner, B. Wellegehausen, and A. Wipf, Inhomogeneous phases in the Gross-Neveu model in $1+1$ dimensions at finite number of flavors, Phys. Rev. D 101, 094512 (2020).

[17] M. Buballa and S. Carignano, Inhomogeneous chiral condensates, Prog. Part. Nucl. Phys. 81, 39 (2015).

[18] T. O. Wehling, E. Şaşığlu, C. Friedrich, A. I. Lichtenstein, M. I. Katsnelson, and S. Blügel, Strength of Effective Coulomb Interactions in Graphene and Graphite, Phys. Rev. Lett. 106, 236805 (2011).

[19] M. Koshino, N. F. Q. Yuan, T. Koretsune, M. Ochi, K. Kuroki, and L. Fu, Maximally Localized Wannier Orbitals and the Extended Hubbard Model for Twisted Bilayer Graphene, Phys. Rev. X 8, 031087 (2018).

[20] S. Ulstrup, M. Schüler, M. Bianchi, F. Fromm, C. Raidel, T. Seyller, T. Wehling, and P. Hofmann, Manifestation of nonlocal electron-electron interaction in graphene, Phys. Rev. B 94, 081403 (2016).

[21] D. K. Efetov and P. Kim, Controlling Electron-Phonon Interactions in Graphene at Ultrahigh Carrier Densities, Phys. Rev. Lett. 105, 256805 (2010).

[22] R. C. Brower, C. Rebbi, and D. Schaich, Hybrid Monte Carlo simulation on the graphene hexagonal lattice, Proc. Sci., Lattice2011 (2011) 056 [arXiv:1204 .5424].

[23] P. V. Buividovich and M. I. Polikarpov, Monte-Carlo study of the electron transport properties of monolayer graphene within the tight-binding model, Phys. Rev. B 86, 245117 (2012).

[24] M. V. Ulybyshev, P. V. Buividovich, M. I. Katsnelson, and M. I. Polikarpov, Monte-Carlo Study of the SemimetalInsulator Phase Transition in Monolayer Graphene with Realistic Inter-Electron Interaction Potential, Phys. Rev. Lett. 111, 056801 (2013).

[25] D. Smith and L. von Smekal, Monte-Carlo simulation of the tight-binding model of graphene with partially screened Coulomb interactions, Phys. Rev. B 89, 195429 (2014).

[26] D. Smith and L. von Smekal, Hybrid Monte-Carlo simulation of interacting tight-binding model of graphene, Proc. Sci., Lattice2013 (2013) 048 [arXiv:1311.1130].

[27] D. Smith, M. Koerner, and L. von Smekal, On the semimetal-insulator transition and Lifshitz transition in simulations of mono-layer graphene, Proc. Sci., Lattice2014 (2014) 055 [arXiv:1410.7601].
[28] P. Buividovich, D. Smith, M. Ulybyshev, and L. von Smekal, Interelectron interactions and the RKKY potential between $\mathrm{H}$ adatoms in graphene, Phys. Rev. B 96, 165411 (2017).

[29] P. Buividovich, D. Smith, M. Ulybyshev, and L. von Smekal, Competing order in the fermionic Hubbard model on the hexagonal graphene lattice, Proc. Sci., Lattice2016 (2016) 244 [arXiv:1610.09855]

[30] T. Luu and T. A. Lähde, Quantum Monte Carlo calculations for carbon nanotubes, Phys. Rev. B 93, 155106 (2016).

[31] M. Koerner, D. Smith, P. Buividovich, M. Ulybyshev, and L. von Smekal, Hybrid Monte Carlo study of monolayer graphene with partially screened Coulomb interactions at finite spin density, Phys. Rev. B 96, 195408 (2017).

[32] S. Beyl, F. Goth, and F. F. Assaad, Revisiting the hybrid quantum Monte Carlo method for Hubbard and electron-phonon models, Phys. Rev. B 97, 085144 (2018).

[33] P. Buividovich, D. Smith, M. Ulybyshev, and L. von Smekal, Hybrid-Monte-Carlo study of competing order in the extended fermionic Hubbard model on the hexagonal lattice, Phys. Rev. B 98, 235129 (2018).

[34] P. Buividovich, D. Smith, M. Ulybyshev, and L. von Smekal, Numerical evidence of conformal phase transition in graphene with long-range interactions, Phys. Rev. B 99, 205434 (2019).

[35] J.-L. Wynen, E. Berkowitz, C. Körber, T. A. Lähde, and T. Luu, Avoiding ergodicity problems in lattice discretizations of the Hubbard model, Phys. Rev. B 100, 075141 (2019).

[36] S. Krieg, T. Luu, J. Ostmeyer, P. Papaphilippou, and C. Urbach, Accelerating Hybrid Monte Carlo simulations of the Hubbard model on the hexagonal lattice, Comput. Phys. Commun. 236, 15 (2019).

[37] D. Smith, P. Buividovich, M. Koerner, M. Ulybyshev, and L. von Smekal, Quantum phase transitions on the hexagonal lattice, arXiv:1912.12537.

[38] A. Alexandru, M. Faber, I. Horvath, and K.-F. Liu, Lattice QCD at finite density via a new canonical approach, Phys. Rev. D 72, 114513 (2005).

[39] P. de Forcrand and S. Kratochvila, Finite density QCD with a canonical approach, Nucl. Phys. B, Proc. Suppl. 153, 62 (2006).

[40] A. Nakamura, S. Oka, and Y. Taniguchi, QCD phase transition at real chemical potential with canonical approach, J. High Energy Phys. 02 (2016) 054.

[41] W. Detmold, K. Orginos, and Z. Shi, Lattice QCD at nonzero isospin chemical potential, Phys. Rev. D 86, 054507 (2012).

[42] J. P. Hobson and W. A. Nierenberg, The statistics of a two-dimensional, hexagonal net, Phys. Rev. 89, 662 (1953).

[43] H. Robbins and S. Monro, A stochastic approximation method, Ann. Math. Stat. 22, 400 (1951).

[44] N. Garron and K. Langfeld, Controlling the sign problem in finite density quantum field theory, Eur. Phys. J. C 77, 470 (2017).

[45] M. V. Ulybyshev and S. N. Valgushev, Path integral representation for the Hubbard model with reduced number of Lefschetz thimbles, arXiv:1712.02188. 
[46] R. Blankenbecler, D. J. Scalapino, and R. L. Sugar, Monte Carlo calculations of coupled boson-fermion systems. I, Phys. Rev. D 24, 2278 (1981).

[47] R. T. Scalettar, D. J. Scalapino, and R. L. Sugar, New algorithm for the numerical simulation of fermions, Phys. Rev. B 34, 7911 (1986).

[48] J. Ostmeyer, E. Berkowitz, S. Krieg, T. A. Laehde, T. Luu, and C. Urbach, The semimetal-Mott insulator quantum phase transition of the Hubbard model on the honeycomb lattice, arXiv:2005.11112.

[49] M. Ulybyshev, C. Winterowd, and S. Zafeiropoulos, Lefschetz thimbles decomposition for the Hubbard model on the hexagonal lattice, Phys. Rev. D 101, 014508 (2020).

[50] M. Ulybyshev, C. Winterowd, and S. Zafeiropoulos, Taming the sign problem of the finite density Hubbard model via Lefschetz thimbles, arXiv:1906.02726. 